\title{
S100b Counteracts Neurodegeneration of Rat Cholinergic Neurons in Brain Slices after Oxygen-Glucose Deprivation
}

\author{
Daniela Serbinek, ${ }^{1}$ Celine Ullrich, ${ }^{1}$ Michael Pirchl, ${ }^{1}$ Tanja Hochstrasser, ${ }^{1}$ \\ Rainald Schmidt-Kastner, ${ }^{2}$ and Christian Humpel ${ }^{1}$ \\ ${ }^{1}$ Laboratory of Psychiatry and Exp. Alzheimer's Research, Department of Psychiatry and Psychotherapy, Innsbruck Medical University, \\ Anichstraße 35, 6020 Innsbruck, Austria \\ ${ }^{2}$ C. E. Schmidt College of Biomedical Science, Florida Atlantic University (FAU), Boca Raton, FL 33431, USA
}

Correspondence should be addressed to Christian Humpel, christian.humpel@i-med.ac.at

Received 28 January 2010; Revised 2 March 2010; Accepted 4 March 2010

Academic Editor: Rosario Donato

Copyright ( 92010 Daniela Serbinek et al. This is an open access article distributed under the Creative Commons Attribution License, which permits unrestricted use, distribution, and reproduction in any medium, provided the original work is properly cited.

\begin{abstract}
Alzheimer's disease is a severe chronic neurodegenerative disorder characterized by beta-amyloid plaques, tau pathology, cerebrovascular damage, inflammation, reactive gliosis, and cell death of cholinergic neurons. The aim of the present study is to test whether the glia-derived molecule S100b can counteract neurodegeneration of cholinergic neurons after oxygen-glucose deprivation (OGD) in organotypic brain slices of basal nucleus of Meynert. Our data showed that 3 days of OGD induced a marked decrease of cholinergic neurons ( $60 \%$ of control), which could be counteracted by $50 \mu \mathrm{g} / \mathrm{mL}$ recombinant S100b. The effect was dose and time dependent. Application of nerve growth factor or fibroblast growth factor-2 was less protective. C-fos-like immunoreactivity was enhanced 3 hours after OGD indicating metabolic stress. We conclude that S100b is a potent neuroprotective factor for cholinergic neurons during ischemic events.
\end{abstract}

\section{Introduction}

Alzheimer's disease (AD) is characterized by beta-amyloid plaque depositions, tau pathology, inflammation, cerebrovascular damage, and cell death of cholinergic neurons. The lack of cortical acetylcholine directly correlates with cognitive impairment. Loss of function and degeneration of cholinergic neurons in the basal forebrain has been a central theme in $\mathrm{AD}$ research, leading to cholinergic neuroprotection strategies for therapy [1]. Loss of trophic support by target neurons in the cortex has been considered as one mechanism for cholinergic degeneration [1]. Furthermore, cholinergic neurons show pathological changes such as accumulation of neurofibrillary tangles and axonal abnormalities early during aging and $\mathrm{AD}[2-4]$. Changes of the basal forebrain are already found in the presymptomatic phase of $\mathrm{AD}$ using neuroimaging $[5,6]$. Exogenous trophic factors can support cholinergic neurons in vitro and in vivo and replacement strategies have been under consideration for treating AD. Nerve growth factor (NGF) is so far the most potent trophic factor to protect cholinergic neurons against neurodegeneration [7]. However, other trophic molecules are also of considerable interest, such as, for example, S100b.

$\mathrm{S} 100 \mathrm{~b}$ is a calcium-binding protein predominantly produced in astrocytes [8], but also oligodendrocytes have been shown to express the protein [9]. S100b is released from glial cells and reaches surrounding neurons on which it exerts trophic functions under physiological conditions $[8,10,11]$. Neuroprotection against glutamate toxicity has been shown in neuronal cell culture models [12]. S100b also activates microglial cells and stimulates iNOS or IL1 $\beta$ via the NFkappaB pathway $[13,14]$. RAGE (receptor for advanced glycosylation end products

(AGE)) has also been linked to cellular activation by S100b [15]. S100b has been also linked to AD, because astrocytes are strongly activated in $\mathrm{AD}$ and in the brain and tissue levels of S100b are increased [16]. It was generally thought that the astroglial response serves protective functions, including the increased production of trophic factors and uptake of beta-amyloid peptides, but negative 
aspects of astrocytic activation have also been uncovered [17]. It was postulated that excessive production of S100b could cause damage to neurons in the brain of $\mathrm{AD}$ patients $[16,18]$. In fact, overexpression of human S100b exacerbated brain damage after ischemia-hypoxia $[19,20]$, and arundic acid ameliorated ischemic damage by preventing astrocytic overproduction of S100b [21]. Taken together, at low (nanomolar) concentrations S100b exerts trophic functions on neurons, whereas at high (micromolar) concentrations the protein stimulates cytokine production which in turn may trigger apoptosis [10].

It has been suggested that chronic neurovascular damage and moderate ischemia-hypoxia (silent strokes) may play a role in the progression of $\mathrm{AD}$ [22]. Moderate ischemia and hypoxia can stimulate astrocytes [23]. In the brain afflicted by $\mathrm{AD}$, loss of trophic support coexists with hypoxic conditions stimulating glial cells, resulting in complex interactions that may involve S100b. Ischemic damage of neurons provokes reactions of astrocytes whereby immunohistochemical labeling for S100b is detected in reactive astrocytes and in damaged neurons [24].

The aim of the present study is to explore the role of S100b on cholinergic neurons in our well established organotypic brain slice culture model in which cholinergic basal nucleus of Meynert (nBM) neurons are provided with NGF for two weeks. Slices incubated without NGF display loss of choline acetyltransferase (ChAT) labeling after two weeks [25] which is an indicator of neurodegeneration. Oxygen-glucose deprivation (OGD) of slice cultures has become an established model for "ischemia-like" conditions [26-28]. In this report, we show that prolonged OGD induces loss of ChAT signals of 2-week old nBM neurons and that S100b can protect against the detrimental effects of OGD. No evidence for a negative effect of S100b itself was detected. The present study suggests that S100b plays a protective role for cholinergic neurons undergoing ischemiahypoxia stress.

\section{Materials and Methods}

2.1. Organotypic Brain Slices. Cholinergic neurons in organotypic brain slices were cultured as described by us in detail $[25,29]$. Briefly, the basal nucleus of Meynert of postnatal day 8 (P8) rats was dissected under aseptic conditions, $400 \mu \mathrm{m}$ slices were cut with a tissue chopper (McIlwain, USA), and the slices were placed on $30 \mathrm{~mm}$ Millicell-CM $0.4 \mu \mathrm{m}$ pore membrane culture plate inserts (7-8 slices per membrane). Slices were cultured in 6-well plates at $37^{\circ} \mathrm{C}$ and $5 \% \mathrm{CO}_{2}$ with $1.2 \mathrm{~mL} /$ well of slice medium (50\% MEM/HEPES (Gibco), 25\% heat-inactivated horse serum (Gibco/Lifetech, Austria), 25\% Hanks' solution (Gibco), $2 \mathrm{mM} \mathrm{NaHCO}$ (Merck, Austria), $6.5 \mathrm{mg} / \mathrm{mL}$ glucose (Merck), and $2 \mathrm{mM}$ glutamine (Merck), $\mathrm{pH} 7.2$ ) including $10 \mathrm{ng} / \mathrm{mL}$ nerve growth factor (NGF) for 2 weeks. It is well-established that the $400 \mu \mathrm{m}$ brain slices become thinner during the 2 weeks of incubation and have a thickness of approximately $100 \mu \mathrm{m}$ after 2 weeks. Slices which did not flatten were removed from the experiments. For the experiment the 2-week old slices were cultured for 3 days without NGF and then transferred to different media: (1) medium with normal $33.2 \mathrm{mM}$ glucose or (2) medium with low $(6.15 \mathrm{mM})$ glucose. Recombinant S100b from bovine brain (Calbiochem) was added to the medium at concentrations from $0.1-50 \mu \mathrm{g} / \mathrm{mL}$. Alternatively, $10 \mathrm{ng} / \mathrm{mL}$ NGF or $2 \mathrm{ng} / \mathrm{mL}$ fibroblast growth factor-2 (FGF-2) was added. It has to be noted that slices could not be cultured in medium without any glucose, because then slices become shrunken and cannot be evaluated.

2.2. Oxygen-Glucose Deprivation (OGD). Slices in the wells (with low glucose) were transferred to a Modular Incubator Hypoxia Chamber (MIC-101, Billups-Rothenberg, Inc., Del Mar, CA, USA) connected to a flow meter. The chamber was sealed and a mixture of $95 \% \mathrm{~N}_{2} / 5 \% \mathrm{CO}_{2}$ was flushed at a flow rate of $25 \mathrm{~L} / \mathrm{min}$ for 5 minutes. The in- and outports of the chamber were closed, and the closed air tight system was placed at $37^{\circ} \mathrm{C}$ in an incubator for 1,2 , or 3 days. Measurements by the manufacturer indicate that the $\mathrm{pO}_{2}$ reached a nadir of $35 \mathrm{~mm} \mathrm{Hg}$ after $6 \mathrm{hr}$. At the end of the experiment the outlet port was slowly opened, the chamber opened, and the culture wells taken out and slices fixed for 3 hours with $4 \%$ paraformaldehyde at $4^{\circ} \mathrm{C}$. Control slices were kept at normal $5 \% \mathrm{CO}_{2} /$ air conditions in the incubator at $37^{\circ} \mathrm{C}$.

2.3. Immunohistochemistry. Immunohistochemistry using the avidin-biotin technique was performed to detect cholinergic neurons as described [30, 31]. All incubations for immunohistochemistry were performed free-floating at $4^{\circ} \mathrm{C}$ for 2 days including $0.1 \%$ Triton, which allows good penetration of the antibody into the slices from both sides. Fixed slices were washed for 30 minutes with $0.1 \%$ Triton/PBS (TPBS) at room temperature and pretreated for 20 minutes with $5 \%$ methanol/1\% $\mathrm{H}_{2} \mathrm{O}_{2} / \mathrm{PBS}$. Then the slices were rinsed three times for 10 minutes with PBS, blocked with $20 \%$ horse serum $/ 0.2 \%$ BSA/T-PBS, and then incubated with the primary antibody against ChAT (1:750, Millipore) or c-fos $(1: 1000$, Santa Cruz) in $0.2 \%$ BSA/T-PBS for 2 days at $4^{\circ} \mathrm{C}$. Slices were washed and incubated with secondary biotinylated anti-goat (ChAT) or anti-rabbit (c-fos) antibody ( $1: 200$, Vector Laboratories), for 1 hour at room temperature. After rinsing three times in PBS, slices were incubated in avidin-biotin complex solution (ABC; Elite Standard PK 6100 , Vector Laboratories) for $1 \mathrm{hr}$, then washed three times in $50 \mathrm{mM}$ Tris-buffered saline (TBS), and the signal was detected using $0.5 \mathrm{mg} / \mathrm{mL} 3,3^{\prime}$ diaminobenzidine (DAB) in TBS with $0.003 \% \mathrm{H}_{2} \mathrm{O}_{2}$ as substrate. Slices were then rinsed in PBS and mounted on gelatine-coated glass slides.

2.4. Quantitative Analysis and Statistics. All neuronal counts were based on individual sections and show total number of neurons per slices. The number of microscopically detectable immunoreactive $\mathrm{ChAT}^{+}$neurons was counted in the whole slice visualized under a $20 \times$ objective by an investigator blinded to the treatment code. Multistatistical analysis was obtained by one-way ANOVA, followed by a subsequent 
Fisher PLSD posthoc test by comparing controls against the respective treatments, where $P<.05$ represents statistical significance.

\section{Results}

When brain slices were cultured for 2 weeks in medium with normal glucose levels containing $10 \mathrm{ng} / \mathrm{mL}$ NGF, approximately 140 cholinergic nBM neurons/slice were detectable (Table 1; Figures 1(a) and 1(c)). This number did not change when slices were incubated without NGF for further 3 days (Table 1). Reduction of glucose to $6 \mathrm{mM}$ (glucose deprivation) did not significantly change the number of cholinergic neurons after 1-2-3 days of incubation (Table 1). Slices exposed to hypoxia at normal (high) glucose did not show a reduced number of $\mathrm{ChAT}^{+}$neurons after 1-23 days of incubation (Table 1). However, when slices were incubated in low glucose and hypoxia (oxygen-glucose deprivation, OGD), the number of $\mathrm{ChAT}^{+}$neurons significantly decreased to about 70 neurons/slice after 3 days but not after 1 or 2 days of incubation (Table 1; Figure 1(b)).

In order to test whether S100b counteracts degeneration of cholinergic neurons caused by OGD for 3 days, slices were incubated with $50 \mu \mathrm{g} / \mathrm{mL}$ of S100b during the exposure. S100b counteracted the decrease of $\mathrm{ChAT}^{+}$neurons after 3 days of OGD (Table 1). By comparison NGF also counteracted loss of $\mathrm{ChAT}^{+}$neurons but was less potent (Table 1). FGF-2 only slightly counteracted the OGD effect (Table 1). Incubation of slices with S100b alone did not exert any toxic effect on ChAT expression in cholinergic neurons (Table 1).

In order to verify stressful effects of OGD on neurons, slices were stained for c-fos immunoreactivity at 3 and 24 hours of OGD (Figures 1(d)-1(f)). In controls without OGD, immunohistochemistry showed very few $\mathrm{c}$-fos positive nuclei (Figure 1(d)). The number and intensity of c-fos positive nuclei significantly $(P<.001)$ increased at 3 hours of OGD (Figure 1(e) and 1(f)) and thereafter returned to control levels at 24 hours of OGD.

\section{Discussion}

In the present study we show that cholinergic neurons of the basal nucleus of Meynert undergo neurodegeneration when exposed during 3 days of oxygen-glucose deprivation in a slice culture model. This neurodegeneration could be counteracted by recombinant $\mathrm{S100b}$ which is an endogenous glial protein.

4.1. Cholinergic Neurons in Brain Slices. The cholinergic neurons of the basal forebrain are a functionally homogeneous population, localized in the medial septum/diagonal band of Broca and basal nucleus of Meynert (nBM), provide the major cholinergic innervation to the hippocampus/amygdala and neocortex, respectively, and play a crucial role in cognition and memory. In neurodegenerative disorders such as $\mathrm{AD}$, these neurons are dysfunctional and can further degenerate in the progression of the disease [32]. To study the mechanisms involved in neurodegenerative processes,
TABLE 1: Cholinergic neurons after glucose-oxygen deprivation and neuroprotection with S100b.

\begin{tabular}{|c|c|c|c|c|}
\hline & Treatment & $\mathrm{ChAT}^{+}$neurons & p1 & p2 \\
\hline Day 0 & & $136 \pm 17(14)$ & - & \\
\hline \multirow[t]{4}{*}{$+1 d$} & GD & $128 \pm 17(5)$ & ns & \\
\hline & OD & $131 \pm 27(6)$ & ns & \\
\hline & OGD & $113 \pm 22(6)$ & ns & - \\
\hline & $\begin{array}{c}\text { OGD + S100b } \\
(50 \mu \mathrm{g} / \mathrm{mL})\end{array}$ & $121 \pm 34(3)$ & & ns \\
\hline \multirow[t]{4}{*}{$+2 \mathrm{~d}$} & GD & $130 \pm 21(5)$ & ns & \\
\hline & OD & $124 \pm 21(8)$ & ns & \\
\hline & OGD & $110 \pm 25(6)$ & ns & - \\
\hline & $\begin{array}{c}\text { OGD + S100b } \\
(50 \mu \mathrm{g} / \mathrm{mL})\end{array}$ & $138 \pm 25(6)$ & & ns \\
\hline \multirow[t]{10}{*}{$+3 \mathrm{~d}$} & $(-)$ & $138 \pm 10(18)$ & - & \\
\hline & S100b $(50 \mu \mathrm{g} / \mathrm{mL})$ & $139 \pm 18(19)$ & ns & \\
\hline & GD & $122 \pm 16(13)$ & ns & \\
\hline & OD & $135 \pm 12(12)$ & ns & \\
\hline & OGD & $81 \pm 8(28)$ & $* * *$ & - \\
\hline & $\begin{array}{c}\text { OGD + S100b } \\
(50 \mu \mathrm{g} / \mathrm{mL})\end{array}$ & $130 \pm 11(26)$ & ns & $* * *$ \\
\hline & $\begin{array}{l}\text { OGD + S100b } \\
(1 \mu \mathrm{g} / \mathrm{mL})\end{array}$ & $89 \pm 10(14)$ & $* *$ & ns \\
\hline & $\begin{array}{c}\text { OGD + S100b } \\
(0.1 \mu \mathrm{g} / \mathrm{mL})\end{array}$ & $92 \pm 12(14)$ & * & ns \\
\hline & $\begin{array}{c}\text { OGD + FGF-2 } \\
(2 \mathrm{ng} / \mathrm{mL})\end{array}$ & $102 \pm 9(12)$ & $P=.06$ & ns \\
\hline & $\begin{array}{c}\text { OGD + NGF } \\
(10 \mathrm{ng} / \mathrm{mL})\end{array}$ & $94 \pm 12(18)$ & $*$ & ns \\
\hline
\end{tabular}

Brain slices of the nBM were cultured for 2 weeks with $10 \mathrm{ng} / \mathrm{mL}$ nerve growth factor (NGF), then for 3 days without NGF and then treated under oxygen-glucose deprivation (OGD) with or without S100b or NGF or fibroblast growth factor-2 (FGF-2) for 1-3 days. Values are given as mean \pm SEM ( $n$ in parentheses). Statistical analysis was performed by one-way ANOVA with a subsequent Fisher PLSD posthoc test and compared against the untreated control (p1) or against OGD (p2) $\left({ }^{*} P<.05 ;{ }^{* *} P<.01\right.$; ${ }^{* * *} P<.001$; ns not significant). GD, glucose deprivation; OD, oxygen deprivation; OGD, oxygen and glucose deprivation.

as well as neuroprotective strategies, the organotypic brain slice model has been established [25, 33-37]. Detection of cholinergic neurons was performed using the immunohistochemical marker for the enzyme ChAT, which is expressed in the cell bodies and nerve fibers. A decreased number of $\mathrm{ChAT}^{+}$neurons directly correlates with neurodegeneration of cholinergic neurons. In our brain slice model cholinergic $\mathrm{nBM}$ neurons are axotomized and the number of $\mathrm{ChAT}^{+}$neurons is markedly decreased without exogenous NGF. Application of recombinant NGF counteracts this degeneration, displaying a slight neuroprotective effect at a concentration of $>0.1 \mathrm{ng} / \mathrm{mL}$ NGF and full activity at $>1 \mathrm{ng} / \mathrm{mL}$ NGF [37]. Thus, our brain slice model may allow to study the cholinergic phenotype or the degeneration of the majority of cholinergic neurons, which is seen by shrunken neurons and loss of nerve fibers and a decrease of the ChAT ${ }^{+}$ immunoreactivity. In order to test whether S100b exerts 


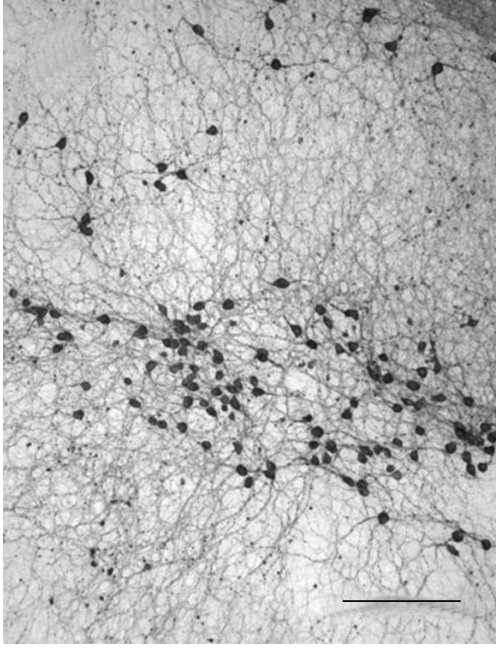

(a)

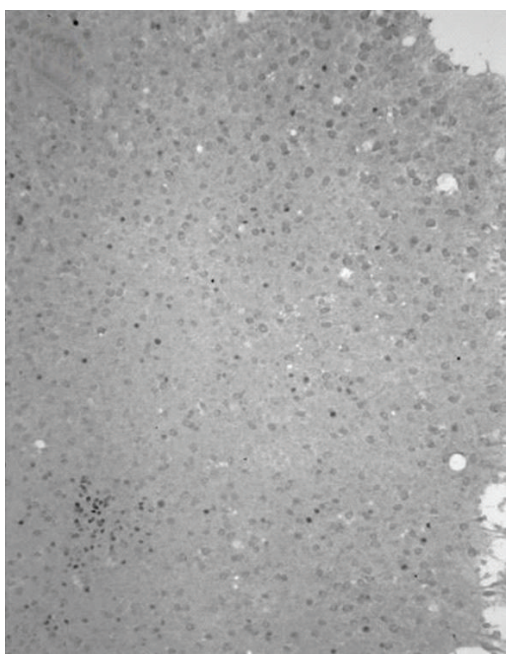

(d)

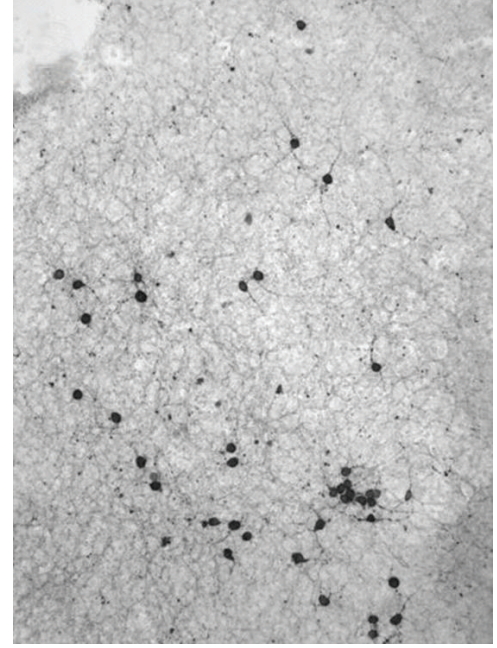

(b)

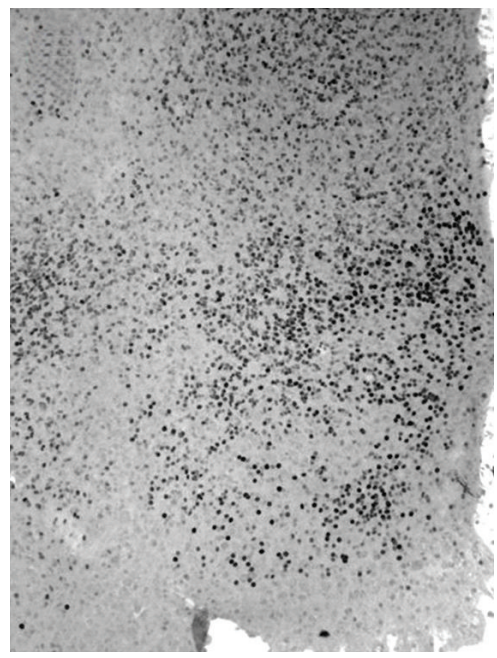

(e)

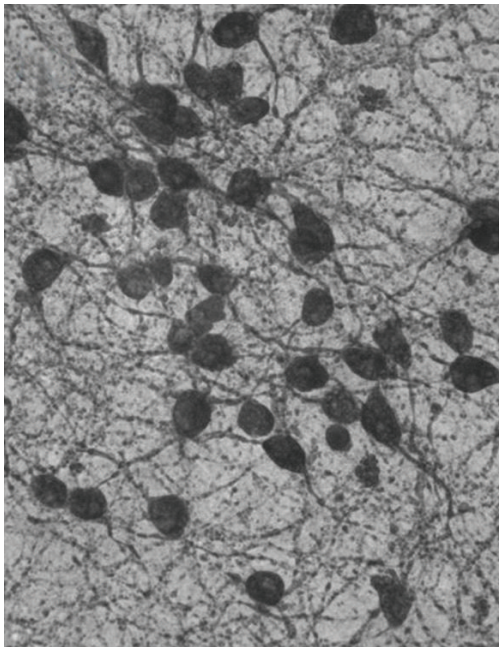

(c)

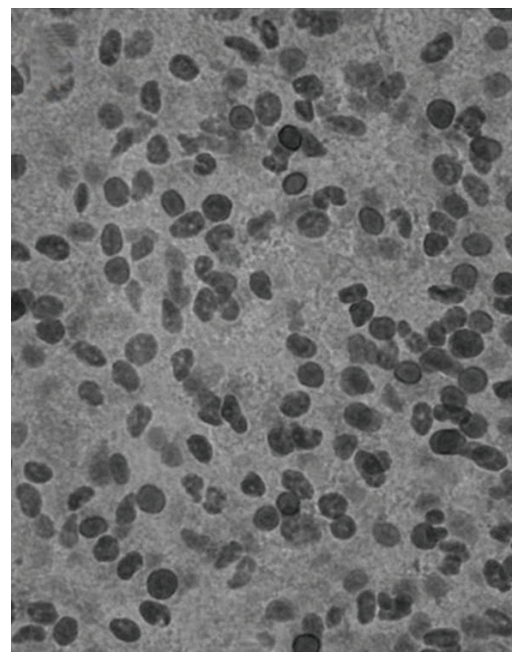

(f)

FIGURE 1: Immunohistochemistry for cholinergic choline acetyltransferase (ChAT) positive neurons (a)-(c) and c-fos immunoreactive nuclei (d)-(f) in organotypic brain slices of the basal nucleus of Meynert. Slices show control stainings before ((a), (c), (d)), or 3 days (ChAT ${ }^{+}$, (b)) or 3 hours (c-fos+, (e) and (f)) after oxygen-glucose deprivation. Scale bar in $A=300 \mu \mathrm{m}((\mathrm{a}),(\mathrm{b}),(\mathrm{d})$, and (e)) and $75 \mu \mathrm{m}((\mathrm{c})$ and (f)).

toxicity on cholinergic neurons, 2 -week slices were exposed to a relatively high dose of $50 \mu \mathrm{g} / \mathrm{mL}(2.38 \mu \mathrm{M})$ of S100b for 3 days, but S100b did not affect the cholinergic neurons in our slice model. It is unlikely that a toxic effect would have shown with short exposures of 1 or 2 days.

4.2. Oxygen-Glucose Deprivation as a Model of Ischemia. The OGD model in slice cultures has been widely used to study "ischemia-like" conditions [26-28]. Most of the studies have used a strong and transient insult in slice cultures of the hippocampus to model the selective vulnerability to ischemia that is observed in vivo after cardiac arrest [26]. Neuronal damage is typically evaluated by dye-uptake and imaging techniques to quantify treatment effects [28]. The present model used a mild and more prolonged OGD impact (i.e., 1-3 d) to simulate the blood flow disturbances in the brain afflicted by vascular changes in AD. The evaluation was focused on the cholinergic neurons in the $\mathrm{nBM}$ using a high-resolution approach with neuronal counting. OGD in slice culture eliminates the influence of blood flow, which otherwise is a complex variable in animal models of brain ischemia. The observation that neurons are more vulnerable to ischemia (= combined loss of oxygen and glucose) than to either hypoglycemia or hypoxia alone has been made in multiple experimental conditions and probably applies to the clinical situation as well. One has to recognize that experimental OGD induces strong reductions but not total removal of glucose and/or oxygen. Since the slices are a stable system, the effects of OGD alone are very weak 
after 3 days. We observed reductions of cholinergic neurons but no widespread cell damage. In the case of glucose deprivation (GD), endogenous stores are not completely exhausted within three days and alternative metabolites may be used. We have data showing that glucose reduction alone for 2 weeks markedly reduced cholinergic neurons. Oxygen deprivation (= hypoxia) is tolerated because of anaerobic glycolysis based on glucose stores which last for three days. Oxygen deprivation longer than 3 days is problematic, because the $\mathrm{pH}$ markedly decreases due to lactate production.

It should be noted that vascular networks persist in the slice culture in the absence of blood flow $[35,38]$. The slice model should provide the opportunity to study reactions of vascular cells to ischemia-like conditions in the absence of intraluminal inflammatory cells. This is an important aspect, because endothelial cells can produce potentially damaging factors for cholinergic neurons [36]. In order to show that cellular stress is initiated soon after onset of oxygen-glucose deprivation, we analyzed the prototypic immediate early gene c-fos, which is upregulated within minutes in response to a strong stimulus, including ischemia, and rapidly downregulated in most experimental systems, even if the stimulus persists. For this purpose it was sufficient to show that c-fos was increased at 3 hours.

4.3. S100b Protects Cholinergic Neurons. The present finding that $\mathrm{S} 100 \mathrm{~b}$ protected cholinergic neurons in the slice culture model against OGD supplements previous reports of a protection of hippocampal neurons from glucose deprivation [39]. At low concentrations, S100b exerts multiple positive effects on neurons, including control of protein phosphorylation, regulation of energy metabolism and function of the cytoskeleton $[8,10]$. Autocrine effects on astrocytes have also been described [10]. The role of S100b stimulation of the RAGE receptor in protection remains to be understood [10]. While there are reports of damaging effects of S100b in high concentrations [10], our experimental data do not suggest a negative influence on cholinergic neurons in vitro, at least for 3 days. In our study we tested different concentrations of $0.1-50 \mu \mathrm{g} / \mathrm{mL}$ but only the highest dose of $50 \mu \mathrm{g} / \mathrm{mL}(=2.38 \mu \mathrm{M})$ provided protection against oxygenglucose deprivation. It has been reported that oxygenserum-glucose deprivation induces release of S100b in the nanomolar concentration from astrocytes after 24 hours [11]. In our slice experiments $0.1 \mu \mathrm{g} / \mathrm{mL}$ S100b did not provide protection. Indeed, we assume that in our model of oxygen-glucose deprivation, the intrinsic astrocytes do release $S 100 \mathrm{~b}$ but this may be insufficient to provide rescue of cholinergic neurons in this insult. A 100-fold higher dose was required to provide protection in our model system with oxygen-glucose deprivation but it is difficult to compare concentrations obtained in different experimental systems. Our organotypic brain slice cultures have a thickness of 100$150 \mu \mathrm{m}$ by two weeks after explantation and it seems likely that a higher dose is necessary for diffusion deep into slices, so that concentrations reached at the level of the cell-bodies several tens of microns deep in the slice may be much lower.
4.4. An Expanded Role for Astrocytes in Alzheimer's Disease. Glial cells surrounding the basal forebrain cholinergic neurons are known to provide important paracrine trophic support, including NGF, FGF-2, epidermal growth factor, brain-derived neurotrophic factor, and ciliary neurotrophic factor. S100b could be an additional trophic factor supplied by local glial cells to cholinergic neurons, and theoretically, a decline of S100b production could contribute to cholinergic dysfunction. So far, most studies have focussed on the activation of astrocytes in the $\mathrm{AD}$ brain which are often related to senile plaques [40]. However, a reduction of glial function could be part of the complex pathophysiology of $\mathrm{AD}$ and improving glial function should be protective [17]. It is notable that two of the major risk genes for late-onset $\mathrm{AD}$, that is, APOE and CLU/Clusterin [41], have important functions in astrocytes $[42,43]$, and gene variants could relate to reduced glial functions. Thus, complex interactions between the primary processes in $\mathrm{AD}$ (beta-amyloid plaque depositions, tau pathology), moderate ischemia-hypoxia, and genetic variations of glial reactions can be envisaged. In fact, it has been reported that S100b protects LAN-5 neuroblastoma cells against beta-amyloid-induced neurotoxicity at lower nanomolar doses, while S100b was toxic to LAN-5 cells at micromolar doses [44]. In their study [44] individual cells were exposed directly to S100b, whereas in our model the protein had to diffuse into the slices. In fact, neurons or glial cells in isolation on culture dishes do not have protection from surrounding cells and are exposed to high levels of radical oxygen species. Our slice model may incorporate these endogenous protective mechanisms. One could argue that our model using a tissue explant is closer to the in vivo situation than cell cultures. Another explanation is also that the recombinant S100b is derived from the bovine gene, while we use rat brain slices, and a mismatch in protein sequence and/or altered dimeric/monomeric forms may contribute to a lower functional efficiency on rat brain S100b sensitive receptors.

In conclusion the present study shows that 3 days of oxygen-glucose deprivation induced a marked decrease of cholinergic neurons, which could be counteracted by S100b. We conclude that $S 100 \mathrm{~b}$ is a potent neuroprotective factor for cholinergic neurons after ischemic events.

\section{Abbreviations}

AD: Alzheimer's disease

ChAT: Choline acetyltransferase

nBM: Basal nucleus of Meynert

NGF: Nerve growth factor

OGD: Oxygen-glucose deprivation.

\section{Acknowledgments}

This study was supported by the Austrian Science Funds (P191220-B05) and the Tyrolean Science Funds. The authors thank Ursula Kirzenberger-Winkler for excellent technical help. They thank Maria Papadopoulou for help with preliminary experiments. 


\section{References}

[1] M. Mesulam, "The cholinergic lesion of Alzheimer's disease: pivotal factor or side show?" Learning and Memory, vol. 11, no. 1, pp. 43-49, 2004.

[2] M. Mesulam, P. Shaw, D. Mash, and S. Weintraub, "Cholinergic nucleus basalis tauopathy emerges early in the aging-MCIAD continuum," Annals of Neurology, vol. 55, no. 6, pp. 815$828,2004$.

[3] K. M. Cullen, G. M. Halliday, K. L. Double, W. S. Brooks, H. Creasey, and G. A. Broe, "Cell loss in the nucleus basalis is related to regional cortical atrophy in Alzheimer's disease," Neuroscience, vol. 78, no. 3, pp. 641-652, 1997.

[4] C. Geula, N. Nagykery, A. Nicholas, and C. K. Wu, "Cholinergic neuronal and axonal abnormalities are present early in aging and in Alzheimer disease," Journal of Neuropathology and Experimental Neurology, vol. 67, no. 4, pp. 309-318, 2008.

[5] A. M. Hall, R. Y. Moore, O. L. Lopez, L. Kuller, and J. T. Becker, "Basal forebrain atrophy is a presymptomatic marker for Alzheimer's disease," Alzheimer's and Dementia, vol. 4, no. 4, pp. 271-279, 2008.

[6] K. Muth, R. Schönmeyer, S. Matura, C. Haenschel, J. Schröder, and J. Pantel, "Mild cognitive impairment in the Elderly is associated with volume loss of the cholinergic basal forebrain region," Biological Psychiatry, vol. 67, no. 6, pp. 588-591, 2009.

[7] R. Levi-Montalcini, "The nerve growth factor 35 years later," Science, vol. 237, no. 4819, pp. 1154-1162, 1987.

[8] R. Donato, "S100: a multigenic family of calcium-modulated proteins of the EF-hand type with intracellular and extracellular functional roles," International Journal of Biochemistry and Cell Biology, vol. 33, no. 7, pp. 637-668, 2001.

[9] J. Steiner, H. G. Bernstein, B. Bogerts, et al., "S100B is expressed in, and released from, OLN-93 oligodendrocytes: influence of serum and glucose deprivation," Neuroscience, vol. 154, no. 2, pp. 496-503, 2008.

[10] M. Rothermundt, M. Peters, J. H. Prehn, and V. Arolt, "S100B in brain damage and neurodegeneration," Microscopy Research and Technique, vol. 60, no. 6, pp. 614-632, 2003.

[11] R. Gerlach, G. Demel, H. G. König, et al., "Active secretion of S100B from astrocytes during metabolic stress," Neuroscience, vol. 141, no. 4, pp. 1697-1701, 2006.

[12] B. Ahlemeyer, H. Beier, I. Semkova, C. Schaper, and J. Krieglstein, "S-100 $\beta$ protects cultured neurons against glutamate- and staurosporine-induced damage and is involved in the antiapoptotic action of the $5 \mathrm{HT}(1 \mathrm{~A})$-receptor agonist, Bay x 3702," Brain Research, vol. 858, no. 1, pp. 121-128, 2000.

[13] A. G. M. Lam, T. Koppal, K. T. Akama, et al., "Mechanism of glial activation by S100B: involvement of the transcription factor NFkB," Neurobiology of Aging, vol. 22, no. 5, pp. 765772, 2001.

[14] L. Liu, Y. Li, L. J. Van Eldik, W. S. T. Griffin, and S. W. Barger, "S100B-induced microglial and neuronal IL-1 expression is mediated by cell type-specific transcription factors," Journal of Neurochemistry, vol. 92, no. 3, pp. 546-553, 2005.

[15] M. A. Hofmann, S. Drury, C. Fu, et al., "RAGE mediates a novel proinflammatory axis: a central cell surface receptor for S100/calgranulin polypeptides," Cell, vol. 97, no. 7, pp. 889901, 1999.

[16] R. E. Mrak and W. S. T. Griffin, "The role of activated astrocytes and of the neurotrophic cytokine S100B in the pathogenesis of Alzheimer's disease," Neurobiology of Aging, vol. 22, no. 6, pp. 915-922, 2001.

[17] D. Farfara, V. Lifshitz, and D. Frenkel, "Neuroprotective and neurotoxic properties of glial cells in the pathogenesis of Alzheimer's disease," Journal of Cellular and Molecular Medicine, vol. 12, no. 3, pp. 762-780, 2008.

[18] J. I. N. G. Sheng, R. E. Mrak, C. R. Rovnaghi, E. W. A. Kozlowska, L. J. Van Eldik, and W. S. T. Griffin, "Human brain $S 100 \beta$ and $S 100 \beta$ mRNA expression increases with age: pathogenic implications for Alzheimer's disease," Neurobiology of Aging, vol. 17, no. 3, pp. 359-363, 1996.

[19] M. S. Wainwright, J. M. Craft, W. S. T. Griffin, et al., "Increased susceptibility of S100B transgenic mice to peinatal hypoxiaischemia," Annals of Neurology, vol. 56, no. 1, pp. 61-67, 2004.

[20] T. Mori, J. U. N. Tan, G. W. Arendash, N. Koyama, Y. Nojima, and T. Town, "Overexpression of human S100B exacerbates brain damage and periinfarct gliosis after permanent focal ischemia," Stroke, vol. 39, no. 7, pp. 2114-2121, 2008.

[21] T. Asano, T. Mori, T. Shimoda, et al., "Arundic acid (ONO2506) ameliorates delayed ischemic brain damage by preventing astrocytic overproduction of S100B," Current Drug Targets-CNS \& Neurological Disorders, vol. 4, no. 2, pp. 127142, 2005.

[22] M. Fotuhi, V. Hachinski, and P. J. Whitehouse, "Changing perspectives regarding late-life dementia," Nature Reviews Neurology, vol. 5, no. 12, pp. 649-658, 2009.

[23] R. Schmidt-Kastner, C. Aguirre-Chen, I. Saul, et al., "Astrocytes react to oligemia in the forebrain induced by chronic bilateral common carotid artery occlusion in rats," Brain Research, vol. 1052, no. 1, pp. 28-39, 2005.

[24] R. Schmidt-Kastner, J. Szymas, and K. A. Hossmann, "Immunohistochemical study of glial reaction and serumprotein extravasation in relation to neuronal damage in rat hippocampus after ischemia," Neuroscience, vol. 38, no. 2, pp. 527-540, 1990.

[25] C. Humpel and C. Weis, "Nerve growth factor and cholinergic CNS neurons studied in organotypic brain slices: implication in Alzheimer's disease?" Journal of Neural Transmission, vol. 62, pp. 253-263, 2002.

[26] A. K. Pringle, F. Iannotti, G. J. C. Wilde, J. E. Chad, P. J. Seeley, and L. E. Sundstrom, "Neuroprotection by both NMDA and non-NMDA receptor antagonists in in vitro ischemia," Brain Research, vol. 755, no. 1, pp. 36-46, 1997.

[27] J. Noraberg, F. R. O. M. Poulsen, M. Blaabjerg, et al., "Organotypic hippocampal slice cultures for studies of brain damage, neuroprotection and neurorepair," Current Drug Targets-CNS \& Neurological Disorders, vol. 4, no. 4, pp. 435452, 2005.

[28] A. P. Raval, H. Bramlett, and M. A. Perez-Pinzon, "Estrogen preconditioning protects the hippocampal CA1 against ischemia," Neuroscience, vol. 141, no. 4, pp. 1721-1730, 2006.

[29] C. Weis, J. Marksteiner, and C. Humpel, "Nerve growth factor and glial cell line-derived neurotrophic factor restore the cholinergic neuronal phenotype in organotypic brain slices of the basal nucleus of Meynert," Neuroscience, vol. 102, no. 1, pp. 129-138, 2001.

[30] B. Zassler and C. Humpel, "Transplantation of NGF secreting primary monocytes counteracts NMDA-induced cell death of rat cholinergic neurons in vivo," Experimental Neurology, vol. 198, no. 2, pp. 391-400, 2006.

[31] C. Humpel, "Basolateral aggregated rat amyloid $\beta(1-42)$ potentiates transmigration of primary rat monocytes through a rat blood-brain barrier," Current Neurovascular Research, vol. 5, no. 3, pp. 185-192, 2008.

[32] C. Hölscher, "Development of $\beta$-amyloid-induced neurodegeneration in Alzheimer's disease and novel neuroprotective strategies," Reviews in the Neurosciences, vol. 16, no. 3, pp. 181212, 2005. 
[33] B. H. Gähwiler, M. Capogna, D. Debanne, R. A. McKinney, and S. M. Thompson, "Organotypic slice cultures: a technique has come of age," Trends in Neurosciences, vol. 20, no. 10, pp. 471-477, 1997.

[34] L. Stoppini, P. A. Buchs, and D. Muller, "A simple method for organotypic cultures of nervous tissue," Journal of Neuroscience Methods, vol. 37, no. 2, pp. 173-182, 1991.

[35] K. V. Moser, R. Schmidt-Kastner, H. Hinterhuber, and C. Humpel, "Brain capillaries and cholinergic neurons persist in organotypic brain slices in the absence of blood flow," European Journal of Neuroscience, vol. 18, no. 1, pp. 85-94, 2003.

[36] K. V. Moser, P. Stöckl, and C. Humpel, "Cholinergic neurons degenerate when exposed to conditioned medium of primary rat brain capillary endothelial cells: counteraction by NGF, MK-801 and inflammation," Experimental Gerontology, vol. 41, no. 6, pp. 609-618, 2006.

[37] B. Zassler, G. Dechant, and C. Humpel, "Urea enhances the nerve growth factor-induced neuroprotective effect on cholinergic neurons in organotypic rat brain slices," Neuroscience, vol. 130, no. 2, pp. 317-323, 2005.

[38] K. Bendfeldt, V. Radojevic, J. Kapfhammer, and C. Nitsch, "Basic fibroblast growth factor modulates density of blood vessels and preserves tight junctions in organotypic cortical cultures of mice: a new in vitro model of the blood-brain barrier," Journal of Neuroscience, vol. 27, no. 12, pp. 32603267, 2007.

[39] S. W. Barger, L. J. Van Eldik, and M. P. Mattson, "S100 $\beta$ protects hippocampal neurons from damage induced by glucose deprivation," Brain Research, vol. 677, no. 1, pp. 167170, 1995.

[40] D. W. Dickson, “The pathogenesis of senile plaques," Journal of Neuropathology and Experimental Neurology, vol. 56, no. 4, pp. 321-339, 1997.

[41] L. Bertram and R. E. Tanzi, "Genome-wide association studies in Alzheimer's disease," Human Molecular Genetics, vol. 18, no. R2, pp. R137-R145, 2009.

[42] M. Koistinaho, S. Lin, X. I. N. Wu, et al., "Apolipoprotein E promotes astrocyte colocalization and degradation of deposited amyloid- $\beta$ peptides," Nature Medicine, vol. 10, no. 7, pp. 719-726, 2004.

[43] E. Törnqvist, L. Liu, H. Aldskogius, H. Von Holst, and M. Svensson, "Complement and clusterin in the injured nervous system," Neurobiology of Aging, vol. 17, no. 5, pp. 695-705, 1996.

[44] R. Businaro, S. Leone, C. Fabrizi, et al., "S100B protects LAN5 neuroblastoma cells against $\mathrm{A} \beta$ amyloid-induced neurotoxicity via RAGE engagement at low doses but increases $\mathrm{A} \beta$ amyloid neurotoxicity at high doses," Journal of Neuroscience Research, vol. 83, no. 5, pp. 897-906, 2006. 


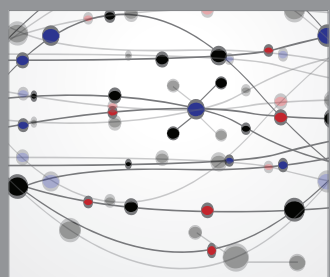

The Scientific World Journal
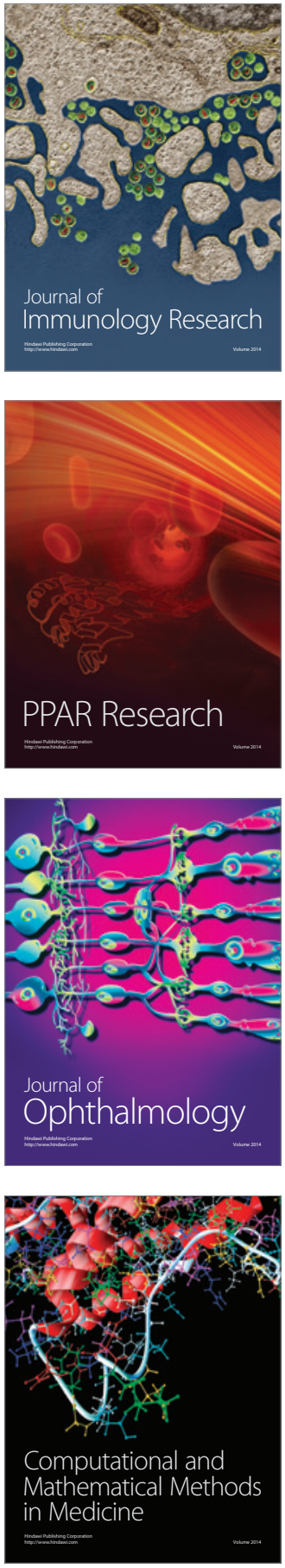

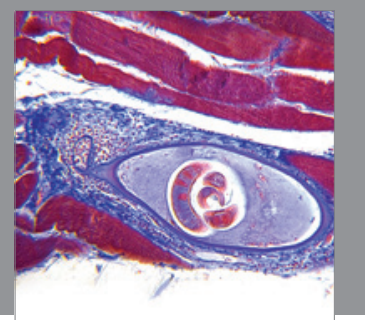

Gastroenterology

Research and Practice
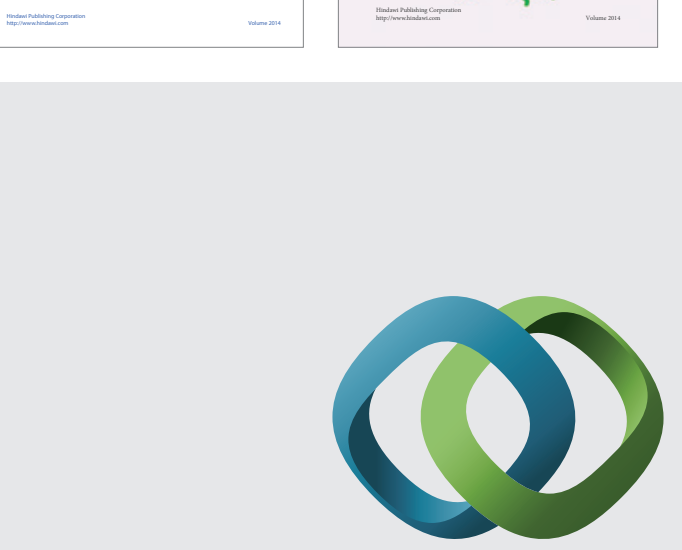

\section{Hindawi}

Submit your manuscripts at

http://www.hindawi.com
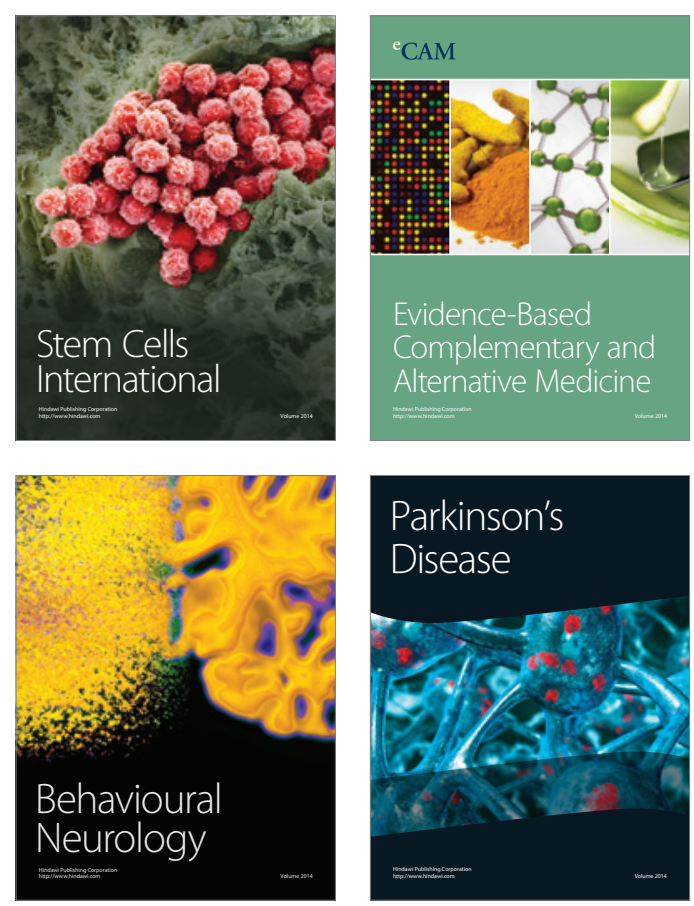

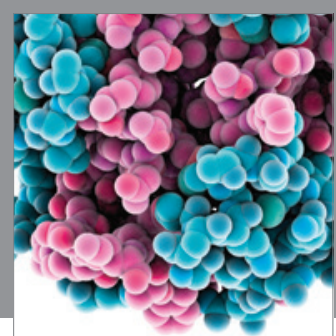

Journal of
Diabetes Research

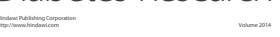

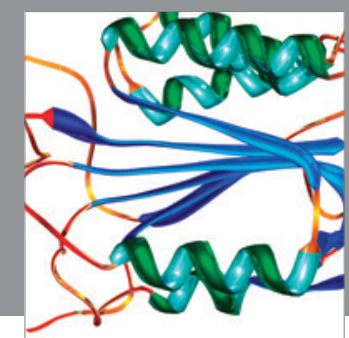

Disease Markers
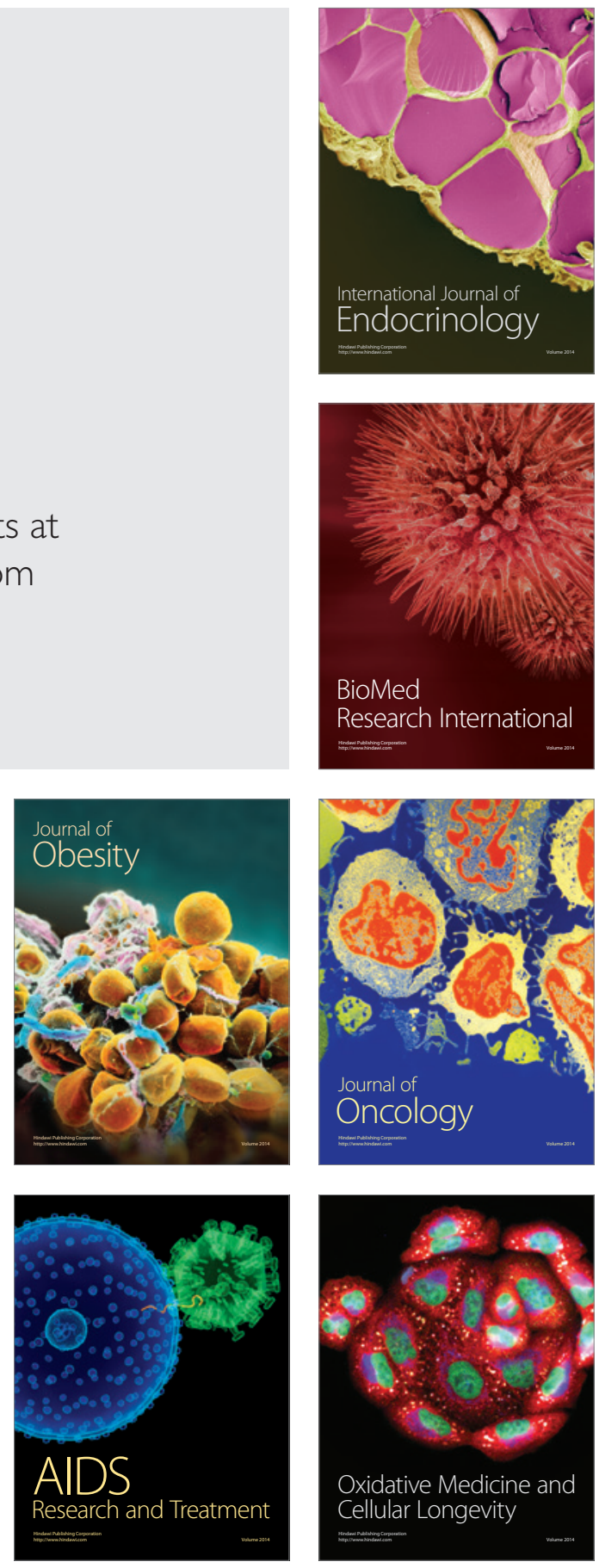